\title{
MARITIME BORDER CONTROL SYSTEM USING GNSS AND GSM
}

\author{
Krishna Kumar \\ Assistant Professor, Vel \\ Tech, Chennai, India
}

\author{
Selvakumar \\ UG Student, Vel Tech, \\ Chennai, India
}

\author{
Mohamed Ashiq \\ UG Student, Vel Tech, \\ Chennai, India
}

\author{
Dhinesh Kumar \\ UG Student, Vel Tech, \\ Chennai, India
}

\begin{abstract}
The major objective of the project is for monitoring the nautical vehicle or vessel during passing the border of the country. Indian fisherman's faces one main problem that is capturing by neighboring countries. The major reason for this problem is that there is no sufficient knowledge about their border position over the sea. We have applied the technologies of GNSS as well as GSM at this system. GNSS stands for Global Navigation Satellite System whereas GPS stands for Global Positioning System. The technology of GNSS is used to find out the navigation or to monitoring the boat's current location accurately. One of the elements of GNSS is called GPS. Mobile communication as well as alerts is utilized through GSM. GSM stands for Global System for Mobile Communication. The directions of latitude as well as longitude are forwarded to microcontroller unit utilizing GNSS. Then, by comparing the current directions of latitude as well as longitude along the pre-defined values the controller unit detects the present location. This technology identifies the nautical border that's why it gives the caution signal to reach fisherman nautical border. In case the fisherman's does not stop the boat we establish a motor driver to stop, hence the boat will stopped automatic. This system is mostly organized for facing the inconvenient of coastal people.
\end{abstract}

Keywords-ARDUINO mega, GNSS (Neo-7M), GSM900, LCD display, Motor driver, Piezoelectric buzzer.

\section{INTRODUCTION}

Almost 18,000 boats from TamilNadu is fishing India-Sri Lanka maritime border. In coastal areas the coastal people livelihoods completely depend upon fishing occupation at sea. If fisherman's passing the border it would be treated into severe offence. The fisherman's frequently cross the maritime border because unaware of the border (Dhivyabharathi and Jeyasingh, 2016). If they cross the border, they are arrested or killed by the marines involved, or they are abducted, their boats are seized by the Coast Guard of the neighboring countries. Continuously the fisherman's lives are danger under such situation. This is one of the main aspects of loss of country economic and also loss in humans. To avoid such inconvenient one new system was created that helps while the fisherman cross the border line will help aware (K.R et al., 2017; Dayana et al., 2018; 2016). We present one system named as advanced SMART Tracking and Nautical Border Alert System utilizing GNSS (spatial dual) presents broad range of navigation as well as timing services along integrated interlocked use of GSM technology.

\section{BLOCK DIAGRAM}

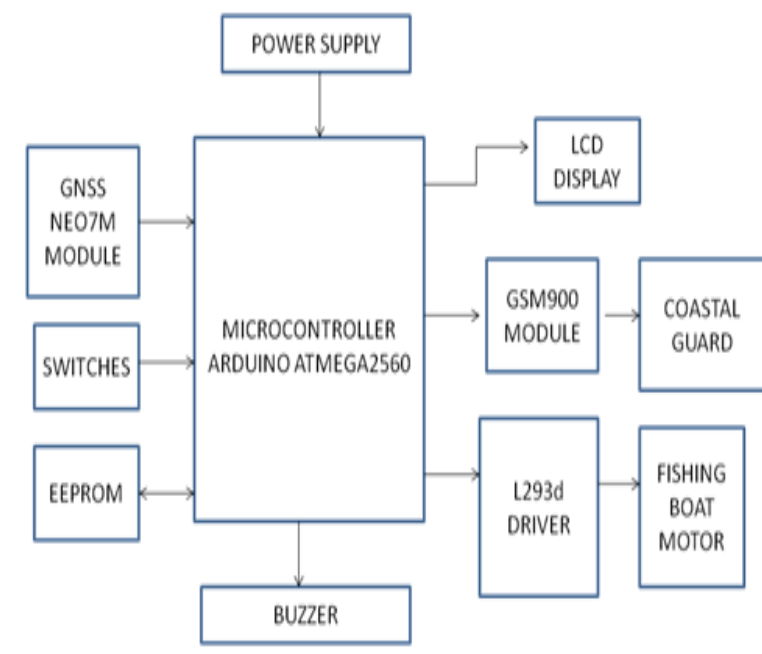

Fig. 1 Block diagram

\section{A. Block Diagram Description}

At this project, suggested embedded system design that is utilized to monitoring as well as positioning of any vehicle through utilizing GNSS as well as GSM. To interface different hardware peripherals the microcontroller is utilized. The present design is an embedded utilization. The present design would continuously tracking on moving boat also inform the boat's status on require. To do so a microcontroller is continuously connected with GSM modem as well as GNSS receiver. GSM modem is utilized to forward the status (latitude as well as longitude) of boat from remote place. GNSS modem provides the data serially that means latitude as 


\section{International Journal of Engineering Applied Sciences and Technology, 2020 Vol. 4, Issue 12, ISSN No. 2455-2143, Pages 358-361 \\ Published Online April 2020 in IJEAST (http://www.ijeast.com)}

well as longitude represents boat's status. GNSS modem outputs several parameters, yet only NMEA data coming out is read and shown on the LCD. From where the boat's status is demanded the same data is forwarded to the mobile on other end. To save the mobile number is utilized EEPROM.

To microcontroller the hardware interfaces are LCD display, GSM modem, GNSS Receiver. RS-232 protocol design is employed to serial communication among modem as well as microcontroller (Sachan, 2012). To convert TTL voltage level to RS-232 voltage levels serial driver IC is used. The systems will automatically sending return reply to the mobile representing boat's status based on latitude as well as longitude when the user's request is forwarded to the number on modem. In general, no invention of recent technology can be implemented without a power source. Hence, in rapid moving world we require deliberate exact power source that is suitable for specific need. Every electronic component is starting from diode to Intel IC's only function to DC supply range from $-+5 \mathrm{v}$ to- $+12 \mathrm{v}$. We use $230 \mathrm{v}-50 \mathrm{~Hz}$ as the cheapest also most commonly available energy source and stepping down, rectifying, filtering as well as voltage regulation. At forth-coming sections it will in brief determined.

\section{PRINCIPLE OF OPERATION}

Suggested for designing one embedded system utilizing GNSS as well as GSM for protect and also positioning the boat at this project. ARDUINO AT MEGA A2560 microcontroller is utilized to interface with multiple hardware devices at this project. The present design is an embedded utilization that continuously tracks the moving boat as well as provides relevant information as the boat moves beyond the defined layer (Wankhade \& Dahad, 2011). To do so, ARDUINO AT MEGA A2560 microcontroller is continuously connected to GSM modem as well as GNSS receiver. From remote place to send the boat's status (latitude as well as longitude) GSM modem is utilized (Pham et al., 2013). GNSS modem is serially providing the data that means by latitude as well as longitude representing boat's status. GNSS modem outputs several parameters, yet only NMEA data coming out is read as well as shown on the LCD. From where the boat's status is demanded the same data is forwarded to the mobile on other end. To save the data obtained through GNSS receiver is utilized EEPROM. To microcontroller the hardware interfaces are LCD display, GSM modem, GNSS Receiver. A MUX is utilized to interface the GSM modem as well as GNSS receiver to the controller (Fleischer et al., 2012). RS-232 protocol design is employed to serial communication among modem as well as microcontroller. To convert TTL voltage level to RS-232 voltage levels serial driver IC is used (Nandaniya et al., 2014). The systems will automatically sending return reply to the mobile representing boat's status based on latitude as well as longitude when the user's request is forwarded to the number on modem.

\section{CIRCUIT DIAGRAM}

This project is mainly used to safety and positioning system, with ARDUINO we could detect the boat location anywhere in world.

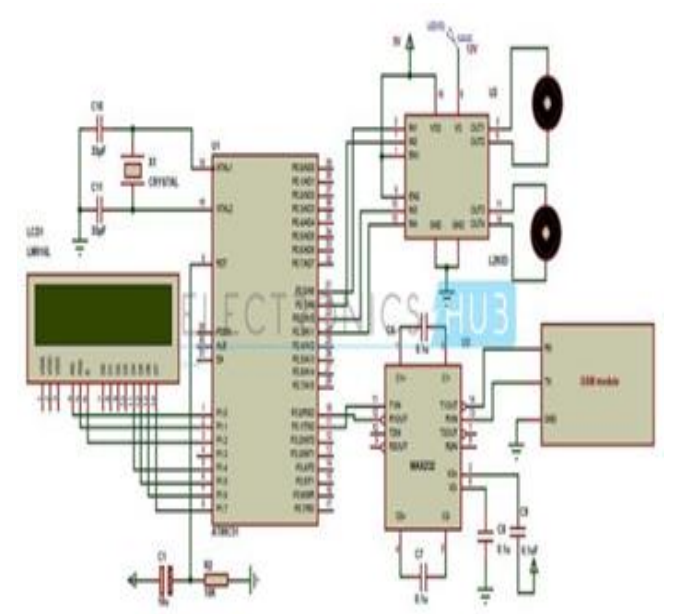

Fig. 2 Circuit Diagram

AT MEGA A2560 micro controller, GNSS receiver, GSM modem, MAX 232, EEPROM, NAND gate and Google maps. Microcontroller used is ARDUINO AT MEGA A2560. The nautical border the area is divided as four different types of zones. They are normal zone, warning zone, near to res. Zone, restricted zone. LCD shows normal zone if the boat is in normal region. Then it is clearly known that the particular boat is at normal region. In case it moves moreover will reaches warning zone, immediately, LCD will display warning zone. Sometimes fisherman did not see display, if he moves moreover, then the boat is entering into close to res Zone, instantly the alarm is operated and boat engine speed is controlled by $50 \%$. If the fisherman has not any sufficient knowledge about the alarm, he moves moreover, when the boat is entering into the restricted zone, the alarm is on and it is by control the flow of fuel then the boat engine will come off position. Code is written at microcontroller's internal memory. For example RAM. It execute the instructions with help of instruction set as well as with help of serial communication it performs into interface among GSM as well as GNSS, also a switch connected to port 1 of ARDUINO AT MEGA A2560. With the help of NAND gate microcontroller switches between GNSS and GSM. GNSS always only transmit the data but GSM also transmit the data as well as obtain the data. GNSS pin TX is linked with microcontroller through NAND gate. GSM pin TX as well as RX are linked with microcontroller through NAND gate. This project is operated in two modes: Manual and Automated. In the Manual mode, the microcontroller verifies "STATUS" as well as sending data whenever user requests. When receives SMS from owner for sending the data RI pin of GSM will be HIGH. Microcontroller found GSM received an SMS decode and 
with the help of AT commands sending information to the owner. In automated mode, microcontroller sending the data to the owner at regular time intervals. Information is latitude and longitude values. Microcontroller does not check for RI interrupt of GSM, directly sends data to the predefined number with help of AT command set. Microcontroller communicates with continuous communication help. Initially it extracts the information from GNSS receiver, with help of GSM modem sending data to the owner in SMS form. To obtain the data from space segment GNSS receiver falcom along 9600 baud rate is utilized (from Satellites), different Satellites GNSS values are forwarded to microcontroller ARDUINO AT MEGA A2560, then such values are processed as well as sent to GSM. GNSS obtains only \$GPRMC values at the processing time. From such values microcontroller extracts only latitude as well as longitude values except time, altitude, satellite name, and authentication and so on. E.g. LAT: 1728:2470 LOG: 7843.3089 GSM modem along baud rate 9600. To mobile communication GSM is the Global system, it performs as SMS receiver as well as SMS sender at this project. Electrically Erasable read only memory is known as EEPROM that saves information temporally stores the GNSS latitude and Longitude Values at regular intervals of time. Whenever user requests to see the information on the kit we can see the information on the LCD display by pressing the display button. Pin 7 acts as read/write of AT24C256 Contains two kinds of power supply one is ac mode and another one is Battery mode. In the battery mode, supplying power to components such as GSM, GNSS as well as Micro control circuitry utilizing a12/3.2A battery. GNSS needs 12v, GSM as well as microcontroller needs $5.3 \mathrm{v}$. We regulate the power among three components with help of regulators. At AC mode, utilized Step-down transformer for convert $230 \mathrm{v}$ AC to $12 \mathrm{v}$, 1Amp Dc and then goes to Bridge circuitry to control ripples in DC signal. The signal is again passed through filter to get pure DC signal and then goes to regulators and to the circuitry.

\section{GNSS SYSTEM}

The expansion of GNSS is Global Navigation Satellite System. It provides reliable positioning, navigation, time services to global users on a series basis at all kind of weather, day and night, on or near Earth with uninterrupted view four or more GNSS satellites (Zaidi and Suddle, 2006; Dow et al., 2009). GNSS comprises three categories: space, control, and user. In the Medium Earth Orbit the space division is made up of 24 to 32 satellites, also includes the boosters needed to launch them as orbit. The Control Segment consists of Master Control Station, Alternate Master Control Station, host of dedicated as well as shared Ground Antennas, Monitor Stations. The Segment of User has hundreds of thousands of U.S. as well as secure allied military users GNSS accurate Service of Positioning and tens of millions of civil, commercial as well as scientific users of the Standard Positioning Service. From space GNSS satellites broadcast signals, which GNSS receivers utilize to given the location of three dimensional (latitude, longitude, as well as altitude) including accurate time.

GNSS mostly utilized help for worldwide navigation, effective tool for map-making, land surveying, commerce, scientific uses, monitoring as well as surveillance, hobbies like geo caching as well as way marking. Further, reference of accurate time is utilized at several utilizations with scientific study of earthquakes, as a synchronization source of time to protocols of cellular network. GNSS has become a major platform for global transportation systems, presenting navigation for aviation, ground as well as nautical operations (Hofmann et al., 2007).

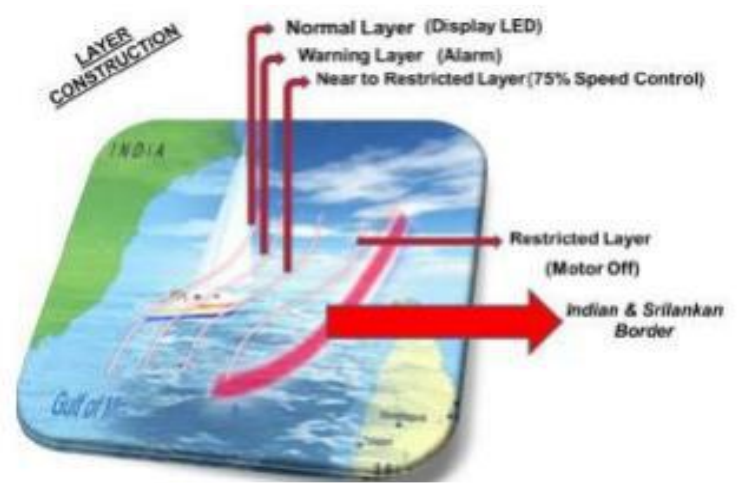

Fig. 3 Layer construction

\section{GNSS TRANSMITTER}

Atomic clocks in a satellite consists of higher stability than 2.1013 [iv]. The fundamental frequency of $10.23 \mathrm{MHz}$ is obtained from the resonant frequency of one of the four atomic clocks on a satellite. To do so, carrier frequency, data frequency, time to generate of pseudo-random noise (PRN) as well as C / A code (course / acquisition code) is obtained from this fundamental frequency. A process called CDMA multiplex (code division multiple access) is utilized since all 28 satellites are transmitting at $1575.42 \mathrm{MHz}$.. Data is sending in terms of DSSS modulation. DSSS stands for Direct Sequence Spread Spectrum Modulation [v]. The generator of C/A code consists of $1023 \mathrm{MHz}$ frequency as well as 1,023 chips period that related to millisecond. The used C/A code (PRN code) that is similar as gold code, hence exhibits the properties of well correlation, it is generated through feedback shift register. The above explained process is indicated as DSSS modulation also in this process $\mathrm{C} / \mathrm{A}$ code plays a key role. Since all satellites are transmitting at the same frequency $(1575.42 \mathrm{MHz}), \mathrm{C} / \mathrm{A}$ code has identities and information that every unique satellite generates. C / A code are an explicit random array of 1023 bits called pseudo-random noise (PRN). 


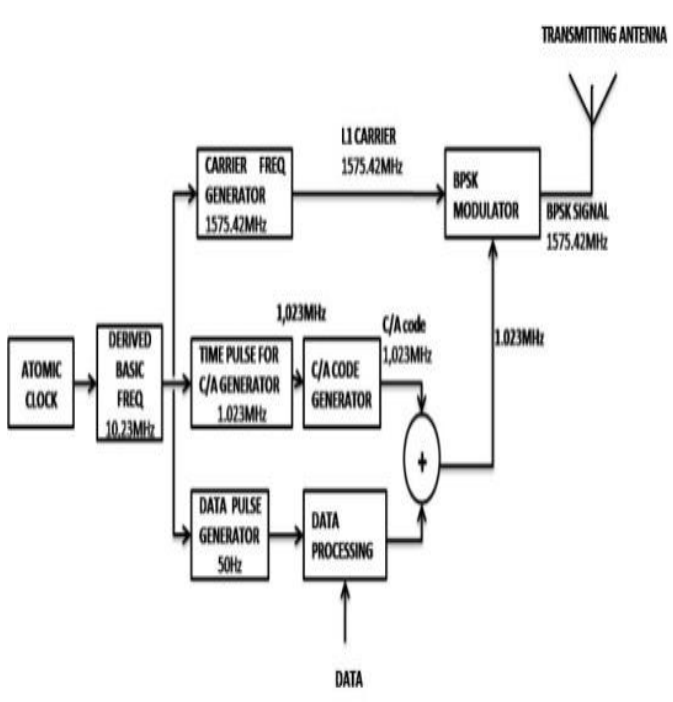

Fig. 4 GNSS Transmitter

\section{CONCLUSION}

Smart monitoring as well as border alert System utilizing GNSS as well as GSM a sample for boat tracking unit can be executed with the help of Google maps, GNSS receiver, GSM modem. Along $10 \mathrm{~m}$ accuracy this system is utilized to protect the boat and also navigate the boat. Through utilizing Google map the positioning is made in latitude as well as longitude form along correct location of place. This system monitor Dayana, M., Vengadeswari, M., and Vinothini, G. (2018). Fishermen Nautical Border Alert System International Journal of Trend in Scientific Research and Development, 2(3), 19711973.

ippdi varum specific boat's location also forwarded the message in SMS form and EEPROM through GSM. The attained data is in latitude as well as longitude form which is used to find the boat on Google maps, moreover the output can be found on the LCD. We require decode the obtained SMS to see it on Google map. At the end, the objective of the project, namely from nautical border save the fishermen as well as navigate the boat with $10 \mathrm{~m}$ accuracy MBR method utilizing is effectively attained. MBR stands for Maritime Border Refuge.

\section{REFERENCE}

[1] Dayana, M.,Vengadeswari, M., and Vinothini, G.(2018). Fishermen Nautical Border Alert System International Journal of Trend in Scientific Research and Development, 2(3), 1971-1973..

[2] Gaming Law Review and Economics. (2016). 20:859868. dio: http://doi.org/10.1089/glre.2016.201011

[3] K. R, R. S, S. S and P. K, (2017). Automatic Border Alert System for Fishermen using GPS and GSM techniques
Indonesian Journal of Electrical Engineering and Computer Science, 7( 1), 84.

[4] Dhivyabharathi,P., and Jeyasingh,Y.(2016) .GPS based Border Alert System using Arduino International Journal of Engineering Research and, 5( 03).

[5] Wankhade, P. P., and Dahad, S. O. (2011). Real time vehicle locking and tracking system using GSM and GPS technology-an anti-theft system. International Journal of Technology And Engineering System (IJTES), 2(3).

[6] Pham, H. D., Drieberg, M., and Nguyen, C. C. (2013). Development of vehicle tracking system using GPS and GSM modem. In 2013 IEEE conference on open systems (ICOS) (pp.89-94)

[7] Sachan, A. (2012). Microcontroller based substation monitoring and control system with gsm modem. IOSR Journal of Electrical and Electronics Engineering, 1(6), 13-21.

[8] Fleischer, P. B., Nelson, A. Y., Sowah, R. A., \& Bremang, A. (2012). Design and development of GPS/GSM based vehicle tracking and alert system for commercial inter-city buses. In 2012 IEEE 4th International Conference on Adaptive Science \& Technology (ICAST) (pp.1-6)

[9] Nandaniya, K., Choksi, V., Patel, Aand \& Potdar, M. B. (2014). Automatic accident alert and safety system using embedded GSM interface. International Journal of Computer Applications, 85(6).

[10] Zaidi, A. S., and Suddle, M. R. (2006). Global navigation satellite systems: a survey. In 2006 international conference on advances in space technologies (pp.84-87)

[11] Dow, J. M., Neilan, R. E., and Rizos, C. (2009). The international GNSS service in a changing landscape of global navigation satellite systems. Journal of geodesy, 83(3-4), 191-198.

[12] Hofmann-Wellenhof, B., Lichtenegger, H., \& Wasle, E. (2007). GNSS-global navigation satellite systems: GPS, GLONASS, Galileo, and more. Springer Science \& Business Media. 